\title{
On Construction of A Control Operator Applied To Conjugate Gradient Method in Solving Continuous Time Linear Regulator Problems with Delay-I
}

\author{
F. M. ADERIBIGBE and K. J. ADEBAYO \\ Department of Mathematical Sciences, Ekiti State University, Ado Ekiti, Nigeria.
}

\begin{abstract}
In this paper, we constructed a control operator, $\boldsymbol{G}$, which enables a Conjugate Gradient Method $(C G M)$ to be employed in solving continuous time linear regulator problems with delay parameter in the state variable. The control operator takes care of any of Mayer's, Lagrange's and Bolza's cost form of linear regulator problems. It is the desire of the authors of this paper that the application of this control operator will further improve on the result of the Conjugate Gradient Method in solving this class of optimal control problem.

Keywords: Control Operator, Optimal Control, Continuous Linear Regulator Problem, Conjugate Gradient Method and Differential Delay State.
\end{abstract}

\section{Introduction}

The performance measure to be minimized is a continuous time linear regulator problem considered by [3] and the Bolza's problem type by [6] and [1] as:

Problem (P1):

$J\left(x, t_{0}, t_{f}, u().\right)=\frac{1}{2} x^{T}\left(t_{f}\right) H x\left(t_{f}\right)+\frac{1}{2} \int_{t_{0}}^{t_{f}}\left\{x^{T}(t) Q(t) x(t)+u^{T}(t) R(t) u(t)\right\} d t$

subject to the differential delay state equation

$\dot{x}(t)=C_{1} x(t)+C_{2} x(t-r)+D u(t), \quad t_{0} \leq t \leq t_{f}$,

$x(t)=h(t),-r \leq t \leq 0$

where $\mathrm{H}$ and $Q(t)$ are real symmetric positive semi-definite $n \times n$ matries. $R(t)$ is a real symmetric positive definite $m \times m$ matrix, the initial time, $t_{0}$, and the final time, $t_{f}$, are specified. $x(t)$ is an $n$-dimensional state vector, $u(t)$ is the $m$-dimensional plant control input vector. $u(t)$ and $x(t)$ are not constrained by any boundaries. $C_{1}, C_{2}$ and $D$ are specified constants which are not necessarily positive, the delay parameter, $r>0$ and $h(t)$ is a given piecewise continuous function which is of exponential order on $[-r, 0]$. If $\mathrm{H}=0,(1.1)$ is called a Lagrange problem, but Mayer problem if $\mathrm{Q}(\mathrm{t})$ and $\mathrm{R}(\mathrm{t})$ are both zero matrices.

According to [2], the controlled differential-delay constraint (1.2) constitutes an important model which has been used variously. Sequel to this, (1.1) can be rewritten as:

$J=\int_{t_{0}}^{t_{f}}\left\{\frac{d}{d t}\left(\frac{1}{2} x^{T}\left(t_{f}\right) H x\left(t_{f}\right)\right)\right\} d t+\frac{1}{2} \int_{t_{0}}^{t_{f}}\left\{x^{T}(t) Q(t) x(t)+u^{T}(t) R(t) u(t)\right\} d t$

$J=\int_{t_{0}}^{t_{f}}\left\{x^{T}(t) H \dot{x}(t)\right\} d t+\frac{1}{2} \int_{t_{0}}^{t_{f}}\left\{x^{T}(t) Q(t) x(t)+u^{T}(t) R(t) u(t)\right\} d t$

$J=\int_{t_{0}}^{t_{f}}\left\{x^{T}(t) H \dot{x}(t)+\frac{1}{2} x^{T}(t) Q(t) x(t)+\frac{1}{2} u^{T}(t) R(t) u(t)\right\} d t$.

As customary with penalty function techniques, constrained problem equations (1.2) and (1.6) may be put into the following equivalent form:

$$
\begin{array}{r}
\begin{array}{l}
\langle z, \boldsymbol{G} z\rangle_{k}=\min _{(x, u)} \int_{t_{0}}^{t_{f}}\left\{x^{T}(t) H \dot{x}(t)+\frac{1}{2} x^{T}(t) Q(t) x(t)+\frac{1}{2} u^{T}(t) R(t) u(t)\right. \\
\left.+\mu\left\|C_{1} x(t)+C_{2} x(t-r)+D u(t)-\dot{x}(t)\right\|^{2}\right\} d t
\end{array} \\
x(s)=h(s) ; s \in[-r, 0]
\end{array}
$$

where $\mu>0$ is the penalty parameter and $\left\|C_{1} x(t)+C_{2} x(t-r)+D u(t)-\dot{x}(t)\right\|^{2}$ is the penalty term. Let us denote by $\tilde{k}$ the product space

$$
\tilde{k}=\mathcal{H}\left[t_{0}, t_{f}\right] \times \ell_{2}\left[t_{0}, t_{f}\right] \times \ell_{2}[-r, 0]
$$

of the Sobolev space $\mathcal{H}\left[t_{0}, t_{f}\right]$ of absolutely continuous function $x($.$) such that, both x($.$) and \dot{x}($.$) are square$ integrable over the finite interval $\left[t_{0}, t_{f}\right]$ and the Hilbert space $\ell_{2}[\alpha, \beta]$ of equivalence classes of real valued functions on $[\alpha, \beta]$ with norm defined by: 
$\|f(.)\|_{\ell_{2}[\alpha, \beta]}=\left(\int_{\alpha}^{\beta}|f(t)|^{2} d t\right)^{\frac{1}{2}}, f(.) \in \ell_{2}[\alpha, \beta]$.

Then, the inner product $\langle., .\rangle_{\tilde{k}}$ on $\tilde{k}$ is given by

$$
\langle., .\rangle_{\tilde{k}}=\langle., .\rangle_{\mathcal{H}\left[t_{0}, t_{f}\right]}+\langle., .\rangle_{\ell_{2}\left[t_{0}, t_{f}\right]}+\langle., .\rangle_{\ell_{2}[-r, 0]} \text {. }
$$

Suppose $z(t) \in \tilde{k}$ denotes ordered triple pair

$$
z^{T}(t)=(x(t), u(t), h(t)) ; x(t) \in \mathcal{H}\left[t_{0}, t_{f}\right], u(t) \in \ell_{2}\left[t_{0}, t_{f}\right] \text { and } h(t) \in \ell_{2}[-r, 0],
$$

then, we seek to determine the operator $\mathbf{G}$ on $\tilde{k}$ such that

$$
\begin{aligned}
\langle z, \boldsymbol{G} z\rangle_{\tilde{k}}=\int_{t_{0}}^{t}\left\{x^{T}(t) H \dot{x}(t)\right. & +\frac{1}{2} x^{T}(t) Q(t) x(t)+\frac{1}{2} u^{T}(t) R(t) u(t) \\
& \left.+\mu\left\|C_{1} x(t)+C_{2} x(t-r)+D u(t)-\dot{x}(t)\right\|^{2}\right\} d t
\end{aligned}
$$

where $\tilde{k}$ is suitably chosen Hilbert space.

The control operator, $\mathbf{G}$, is then utilized in the iterative framework of the CGM in order to arrive at a solution of problem (P1). We provide a recapitulation of the formal CGM in the next section for the sake of completeness.

\section{Conjugate Gradient Method Algorithm}

The Conjugate Gradient Method (CGM) algorithm for iteratively locating the minimum $x^{*}$ of $f(x)$ in $\mathcal{H}$ as described by [4] is as follows:

Step 1: Guess the first element $x_{0} \in \mathcal{H}$ and compute the remaining members of the sequence with the aid of the formulae in the steps 2 through 6.

Step 2: Compute the descent direction $p_{0}=-g_{0}$

Step 3: Set $x_{i+1=} x_{i}+\alpha_{i} p_{i}$; where $\alpha_{i}=\frac{\left\langle g_{i}, g_{i}\right\rangle_{\mathcal{H}}}{\left\langle p_{i}, G p_{i}\right\rangle_{\mathcal{H}}}$

Step 4: Compute $g_{i+1}=g_{i}+\alpha_{i} G p_{i}$

Step 5: Set $p_{i+1}=-g_{i+1}+\beta_{i} p_{i} ; \beta_{i}=\frac{\left\langle g_{i+1}, g_{i+1}\right\rangle_{\mathcal{H}}}{\left\langle g_{i}, g_{i}\right\rangle_{\mathcal{H}}}$

Step 6: If $g_{i}=0$ for some $i$, then, terminate the sequence; else set $i=i+1$ and go to step 3 .

In the iterative steps 2 through 6 above, $p_{i}$ denotes the descent direction at $i$-th step of the algorithm, $\alpha_{i}$, is the step length of the descent sequence $\left\{x_{i}\right\}$ and $g_{i}$ denotes the gradient of $F$ at $x_{i}$. Steps 3, 4 and 5 of the algorithm reveal the crucial role of the linear operator $\mathrm{G}$ in determining the step length of the descent sequence and also in generating a conjugate direction of search. Applicability of the algorithm thus depends solely on the explicit knowledge of the operator G. Generally, for discrete optimization problems, $G$ is readily determined (see [4, pp. 51-53]); and such problem enjoys the beauty of the CGM as a computational scheme since the CGM exhibits quadratic convergence and requires only a little more computation per iteration. [2] opined that these properties make the CGM a fascinating computational technique with a strong appeal for implementation on the digital computer.

However, for the type of constrained continuous linear time regulator problem (P1) discussed in this paper, application of the CGM algorithm as presented is hindered because then, the equivalent of operator $\mathrm{G}$ which satisfies (P1) in this sense of (1.13) is not readily found and construction of such operator is the main aim of this paper. Although, the construction of such similar operator is not new. For instance, in [5] and [2], the authors constructed the control operator for the following related problem respectively as:

\section{Problem (2)}

Subject to

$$
\operatorname{Min}_{(x, u)} \int_{t_{0}}^{t_{f}}\left\{a x^{2}(t)+b u^{2}(t)\right\} d t
$$

$$
\begin{aligned}
& \dot{x}(t)=c x(t)+d u(t), \quad t_{0} \leq t \leq t_{f}, \\
& x\left(t_{0}\right)=x_{0} ;
\end{aligned}
$$

where $\mathrm{a}, \mathrm{b}, \mathrm{c}$ and $\mathrm{d}$ specified constants such that a $>0, \mathrm{~b}>0 ; x_{0}, t_{f}$ are given, $\dot{x}(t)$ denotes the derivative of the state $x($.$) with respect to time, and u($.$) is the control vector.$

Problem (3)

$$
\operatorname{Min}_{(x, u)} \int_{t_{0}}^{t_{f}}\left\{A x^{2}(t)+B u^{2}(t)\right\} d t
$$

Subject to

$$
\begin{aligned}
& \dot{x}(t)=C_{1} x(t)+C_{2} x(t-r)+D u(t), \quad t_{0} \leq t \leq t_{f}, \\
& x(t)=h(t) ; \quad-r \leq t \leq 0
\end{aligned}
$$


where $A, B>0$; the delay parameter $r>0$ and $t_{f}$ are given; $C_{1}, C_{2}$ and $D$ are specified constants which are not necessarily positive and $h(t)$ is a given piecewise continuous function which is of exponential order on $[-r, 0]$. Then for $\mu>0$ there exists a control operator $\mathrm{D}$, with $D: \widetilde{k} \times \widetilde{k} \rightarrow \mathcal{R}$ such that $\langle z(t), D z(t)\rangle_{\tilde{k}}=\int_{t_{0}}^{t_{f}}\left\{A x^{2}(t)+B u^{2}(t)+\mu\left\|\dot{x}(t)-C_{1} x(t)-C_{2} x(t-r)-D u(t)\right\|^{2}\right\} d t$ where $z(t)=(x(t), u(t), h(t))^{T}$. Furthermore, D is explicitly given by:

$$
(D z(t)) \equiv\left(\begin{array}{lll}
D_{11} & D_{12} & D_{13} \\
D_{21} & D_{22} & D_{23} \\
D_{31} & D_{32} & D_{33}
\end{array}\right)\left(\begin{array}{l}
x(t) \\
u(t) \\
h(t)
\end{array}\right)=\left(\begin{array}{l}
\left(D_{11} x\right)(t)+\left(D_{12} u\right)(t)+\left(D_{13} h\right)(t) \\
\left(D_{21} x\right)(t)+\left(D_{22} u\right)(t)+\left(D_{23} h\right)(t) \\
\left(D_{31} x\right)(t)+\left(D_{32} u\right)(t)+\left(D_{33} h\right)(t)
\end{array}\right)
$$

where the composite operators $D_{i j}, 1 \leq i, j \leq 3$ are given in detain in [2].

\section{Main Result}

Our results for problem (P1) are contained in the following theorem:

\subsection{Theorem 1}

Let us denote by $\widetilde{k}$ the product space

$\tilde{k}=W^{1,2}\left[t_{0}, t_{f}\right] \times \ell^{2}\left[t_{0}, t_{f}\right] \times \ell^{2}[-r, 0]$

of the Sobolev space of absolutely continuous function $x($.$) on \left[t_{0}, t_{f}\right]$ and the usual Hilbert space $\ell^{2}\left[t_{0}, t_{f}\right]$ of Lebesgue measurable, real-valued functions which are square integrable on the closed interval $\left.t_{0}, t_{f}\right]$. Then ,for $\mu>0$ there exists a control operator, $\mathrm{G}$, with $G: \tilde{k} \times \tilde{k} \rightarrow \mathcal{R}$ such that

$$
\begin{aligned}
\langle z(t), \mathrm{Gz}(t)\rangle_{\tilde{k}}= & \int_{t_{0}}^{t_{f}}\left\{x^{T}(t) H \dot{x}(t)+\frac{1}{2} x^{T}(t) Q(t) x(t)+\frac{1}{2} u^{T}(t) R(t) u(t)\right. \\
& \left.+\mu\left\|C_{1} x(t)+C_{2} x(t-r)+D u(t)-\dot{x}(t)\right\|^{2}\right\} d t
\end{aligned}
$$

where $z(t)=(x(t), u(t), h(t))^{T}$. Furthermore, $\mathrm{G}$ is explicitly given by:

$$
(\mathrm{G} z(t)) \equiv\left(\begin{array}{lll}
\mathrm{G}_{11} & \mathrm{G}_{12} & \mathrm{G}_{13} \\
\mathrm{G}_{21} & \mathrm{G}_{22} & G_{23} \\
G_{31} & G_{32} & G_{33}
\end{array}\right)\left(\begin{array}{l}
x(t) \\
u(t) \\
h(t)
\end{array}\right)=\left(\begin{array}{l}
\left(G_{11} x\right)(t)+\left(G_{12} u\right)(t)+\left(G_{13} h\right)(t) \\
\left(G_{21} x\right)(t)+\left(G_{22} u\right)(t)+\left(G_{23} h\right)(t) \\
\left(G_{31} x\right)(t)+\left(G_{32} u\right)(t)+\left(G_{33} h\right)(t)
\end{array}\right)
$$

where the composite operators $G_{i j}, 1 \leq i, j \leq 3$ are given as follows:

$$
\begin{aligned}
&\left(G_{11} x(t)\right)=-\mu \dot{x}(0) \operatorname{Sinh}\left(t_{f}\right)+\int_{0}^{t_{f}} \mu \dot{x}(s) \operatorname{Sinh}\left(t_{f}-s\right) d s-\int_{0}^{t_{f}} \operatorname{Sinh}\left(t_{f}-s\right) \\
& {\left[\left(\frac{1}{2} Q+\mu C_{1}^{2}+C_{2}^{2}\right) x(s)+\left(H-2 \mu C_{1}\right) \dot{x}(s)+2 \mu C_{1} C_{2} x(s+r)\right.}\left.-2 \mu C_{2} \dot{x}(s+r)\right] d s+\left[\left(\frac{1}{2} Q+\mu C_{1}^{2}+C_{2}^{2}\right) x(0)+\left(H-2 \mu C_{1}\right) \dot{x}(0)\right. \\
&\left.+2 \mu C_{1} C_{2} x(r)-2 \mu C_{2} \dot{x}(s+r)\right] \operatorname{Cosh}(t)+\frac{\sinh t}{\operatorname{Sinh} t_{f}}\left\{\left(\frac{1}{2} Q+\mu C_{1}^{2}+C_{2}^{2}\right) x\left(t_{f}\right)\right. \\
&+\left(H-2 \mu C_{1}\right) \dot{x}\left(t_{f}\right)+2 \mu C_{1} C_{2} x\left(t_{f}-r\right)-2 \mu C_{2} \dot{x}\left(t_{f}-r\right) \\
&-\left[\left(\frac{1}{2} Q+\mu C_{1}^{2}+C_{2}^{2}\right) x(0)+\left(H-2 \mu C_{1}\right) \dot{x}(0)+2 \mu C_{1} C_{2} x(r)\right. \\
&\left.-2 \mu C_{2} \dot{x}(r)\right] \operatorname{Cosh}\left(t_{f}\right)+\int_{0}^{t_{f}} \operatorname{Sinh}\left(t_{f}-s\right)\left[\left(\frac{1}{2} Q+\mu C_{1}^{2}+C_{2}^{2}\right) x(s)\right. \\
&\left.+\left(H-2 \mu C_{1}\right) \dot{x}(s)+2 \mu C_{1} C_{2} x(s+r)-2 \mu C_{2} \dot{x}(s+r)\right] d s \\
&\left.+\mu \dot{x}(0) \operatorname{Sinh}\left(t_{f}\right)-\int_{0}^{t_{f}} \mu \dot{x}(s) \operatorname{Cosh}\left(t_{f}-s\right) d s\right\} ; 0 \leq t \leq t_{f} \\
&\left(G_{21} x(t)\right)=- 2 \mu \operatorname{Dx}(t) ; 0 \leq t \leq t_{f} \\
&\left(G_{31} x(t)\right)=2 \mu C_{1} C_{2} x(t+r)-2 \mu C_{2} \dot{x}(t+r)+\mu C_{2}^{2} x(t) ;-r \leq t \leq 0 \\
&\left(G_{12} u(t)\right)=2 \mu \operatorname{Du}(0) \operatorname{Sinh}\left(t_{f}\right)+\int_{0}^{t_{f}} 2 \mu D u(s) \operatorname{Cosh}\left(t_{f}-s\right) d s \\
& \quad-\int_{0}^{t_{f}} \operatorname{Sinh}\left(t_{f}-s\right)\left[2 \mu C_{1} u(s)+2 \mu C_{2} D u(s+r)\right] d s \\
& \quad+\left[2 \mu C_{1} D u(0)+2 \mu C_{2} D u(r)\right] \operatorname{Cosh}(t) \\
& \quad+\frac{\operatorname{Sinh}(t)}{\operatorname{Sinh}\left(t_{f}\right)}\left\{2 \mu C_{1} D u\left(t_{f}\right)+2 \mu C_{2} \operatorname{Du}\left(t_{f}-r\right)-2 \mu C_{1} D u(0)\right.
\end{aligned}
$$




$$
\begin{aligned}
& -2 \mu C_{2} D u(r)+2 \mu D u(o) \operatorname{Sinh}\left(t_{f}\right)-\int_{0}^{t_{f}} 2 \mu D u(s) \operatorname{Cosh}\left(t_{f}-s\right) d s \\
& +\int_{0}^{t_{f}-r} \operatorname{Sinh}\left(t_{f}-r-s\right)\left[2 \mu C_{1} D u(s)+2 \mu C_{2} D u(s+r)\right] d s ; 0 \leq t \leq t_{f} \\
& \left(G_{22} u(t)\right)=\frac{1}{2} R u(t)+\mu D^{2} u(t) ; 0 \leq t \leq t_{f} \\
& \left(G_{32} u(t)\right)=2 \mu C_{2} D u(t+r) \quad ;-r \leq t \leq 0 \\
& \left(G_{13} h(t)\right)=2 \mu C_{2} h(0) \operatorname{Sinh}(r)-\int_{0}^{r} \operatorname{Cosh}(r-\tau)\left[2 \mu C_{2} h(0)\right] d \tau \\
& -\int_{0}^{r} \operatorname{Sinh}(r-\tau)\left[2 \mu C_{1} C_{2} h(\tau)\right] d \tau+\frac{\operatorname{Sinh}(t)}{\operatorname{Sinh}\left(t_{f}\right)}\left\{2 \mu C_{1} C_{2} h(r)\right. \\
& -2 \mu C_{1} C_{2} h(0) \operatorname{Cosh}(r)-2 \mu C_{2} h(0) \operatorname{Sinh}(r)+\int_{0}^{r} \operatorname{Cosh}(r-\tau)\left[2 \mu C_{2} h(\tau)\right] d \tau \\
& \left.+\int_{0}^{r} \operatorname{Sinh}(r-\tau)\left[2 \mu C_{1} C_{2} h(r)\right] d \tau\right\}+2 \mu C_{1} C_{2} h(0) \operatorname{Cosh}(t) ; 0 \leq t \leq r \\
& \left(G_{13} h(t)\right)=0 ; \quad r=0 \\
& \left(G_{23} h(t)\right)=\left\{\begin{array}{ll}
2 \mu C_{2} D h(s) ; & 0 \leq s \leq r \\
0 ; & r=0
\end{array}\right\} \\
& \left(G_{33} h(t)\right)=\left\{\begin{array}{ll}
\mu C_{2} h(t) ; & 0 \leq s \leq r \\
0 ; & r=0
\end{array}\right\}
\end{aligned}
$$

\section{Proof of Theorem 3.1}

Towards the proof of the above theorem, we need the following fundamentals:

\subsection{Lemma 1:}

Let $n \geq 0$ be an integer and suppose $\mathcal{R}^{n}(0, T)$ denotes the space of all real-valued functions $y(t), 0 \leq t \leq T$ which are n-times continuously differentiable on $[0, T] \subset \mathcal{R}$ with the norm $\|y\|_{n}$ given by $\|y\|_{n}=\sum_{i=0}^{n} \max _{0 \leq t \leq T}\left|y^{(i)}(t)\right|$,

where $\left|y^{(i)}(t)\right|$ denotes the $i$-th derivative of $y(t)$. Then if $\alpha(t)$ and $\beta(t)$ are continuous in $[a, b]$ and if $\int_{a}^{b}[\alpha(t) y(t)+\beta(t) \dot{y}(t)] d t=0$ for every function $g(t) \in \mathcal{R}^{1}(a, b)$ such that $g(a)=g(b)=0$, then $\beta(t)$ is differentiable and $\frac{d}{d t}(\beta(t))=\alpha(t)$ for all $t \in[a, b]$.

\section{Proof:}

For our subsequent development we shall associate with the right hand side of (1.7) the functional $R_{\mu}\left(z_{1}, z_{2}\right)$ defined by

$$
\begin{aligned}
R_{\mu}\left(z_{1}, z_{2}\right) & =\int_{0}^{t}\left\{\frac{1}{2} x_{1}(t) Q x_{2}(t)+\frac{1}{2} u_{1}(t) R u_{2}(t)+x_{1}(t) H \dot{x}_{2}(t)+\mu C_{1}^{2} x_{1}(t) x_{2}(t)\right. \\
& +\mu C_{1} C_{2} x_{1}(t) x_{2}(t-r)+\mu C_{1} D x_{1}(t) u_{2}(t)-\mu C_{1} x_{1}(t) \dot{x}_{2}(t)+\mu C_{1} C_{2} x_{1}(t) x_{2}(t-r) \\
& +\mu C_{2}^{2} x_{1}(t-r) x_{2}(t-r)+\mu C_{2} D x_{1}(t-r) u_{2}(t)-\mu C_{2} x_{1}(t-r) \dot{x}_{2}(t) \\
& +\mu C_{1} D x_{1}(t) u_{2}(t)+\mu C_{2} D x_{1}(t-r) u_{2}(t)+\mu D^{2} u_{1}(t) u_{2}(t)-\mu D u_{1}(t) \dot{x}_{2}(t) \\
& \left.-\mu C_{1} x_{1}(t) \dot{x}_{2}(t)-\mu C_{2} x_{1}(t-r) \dot{x}_{2}(t)-\mu D u_{1}(t) \dot{x}_{2}(t)+\mu \dot{x}_{1}(t) \dot{x}_{2}(t)\right\} d t
\end{aligned}
$$

where $z_{1}^{T}=\left(x_{1}(t), u_{1}(t), h_{1}(t)\right), z_{2}^{T}=\left(x_{2}(t), u_{2}(t), h_{2}(t)\right)$ are the ordered triple pair which belong to the space $\tilde{k}$ defined by (3.1). It follows that; the form (3.14) is equivalent to (3.2) under the equivalent relationships:

$$
\left\{\begin{array}{cc}
x_{1}(t) \equiv x_{2}(t)=x(t), & 0 \geq t \geq t_{f} \\
\dot{x}_{1}(t) \equiv \dot{x}_{2}(t)=\dot{x}(t), & 0 \geq t \geq t_{f}, \\
u_{1}(t) \equiv u_{2}(t)=u(t), & 0 \geq t \geq t_{f} \\
h_{1}(t) \equiv h_{2}(t)=h(t), & -r \geq t \geq 0 .
\end{array}\right\}
$$

For proof, see [7]. We then have the following proposition: 


\subsection{Proposition 1:}

$R_{\mu}\left(z_{1}, z_{2}\right)$ is a bounded, bilinear, self-adjoint form on $\tilde{k}$.

Proof:

Bilinearity and self-adjointness of $R_{\mu}\left(z_{1}, z_{2}\right)$ is clear from its definition; and its boundedness follows from the fact that $z_{i}(t)=\left(x_{i}(t), u_{i}(t), h_{i}(t)\right)^{T}, i=1,2$ is bounded.

\subsection{Remarks:}

By virtue of proposition above and a consequence of the Reiess representation theorem on Hilbert spaces [8], it follows that $R_{\mu}\left(z_{1}, z_{2}\right)$ induces a uniquely determined, bounded linear operator $\mathrm{G}$ say on $\tilde{k}$ with the representation

$$
R_{\mu}\left(z_{1}, z_{2}\right)=\left\langle G z_{1}, z_{2}\right\rangle_{\tilde{k}}=\left\langle z_{1}, G z_{2}\right\rangle_{\tilde{k}}=R_{\mu}\left(z_{1}, z_{2}\right)
$$

Where it is clear that $\mathrm{G}$ is also self-adjoint on $\tilde{k}$ since $R_{\mu}\left(z_{1}, z_{2}\right)$ is.

The prescribed initial function $h(t)$ is linearly related to the delay term $x(t-r)$ in the sense that, for $t \in\left[0, t_{f}\right]$,

$$
x(t-r)=x(s)=\left\{\begin{array}{l}
h(s) ; s \in[-r, 0] \\
x(s) ; s \in\left[0, t_{f}-r\right.
\end{array}\right\}, h(0)=x(0) .
$$

It follows from above that, when $h(t) \equiv 0$, then, $x(t-r)=x(t)$.

Let us now consider the equivalence

$\left\langle z_{1}, G z_{2}\right\rangle=R \mu\left\langle z_{1}, z_{2}\right\rangle$

This is convenient for our subsequent developments.

Then, let $x_{2}(t)=\left(x_{2}(t), u_{2}(t), h_{2}(t)\right)^{T}$, then we can write

$\left(\mathrm{Gz}_{2}(\mathrm{t})\right) \equiv\left(\begin{array}{lll}\mathrm{G}_{11} & \mathrm{G}_{12} & \mathrm{G}_{13} \\ \mathrm{G}_{21} & \mathrm{G}_{22} & G_{23} \\ G_{31} & G_{32} & G_{33}\end{array}\right)\left(\begin{array}{l}x_{2}(t) \\ u_{2}(t) \\ h_{2}(t)\end{array}\right)=\left(\begin{array}{l}\left(G_{11} x_{2}\right)(t)+\left(G_{12} u_{2}\right)(t)+\left(G_{13} h_{2}\right)(t) \\ \left(G_{21} x_{2}\right)(t)+\left(G_{22} u_{2}\right)(t)+\left(G_{23} h_{2}\right)(t) \\ \left(G_{31} x_{2}\right)(t)+\left(G_{32} u_{2}\right)(t)+\left(G_{33} h_{2}\right)(t)\end{array}\right)$

On setting $h_{2}(t) \equiv u_{2}(t)=0$; we obtain

$$
\left(\mathrm{Gz} z_{2}(\mathrm{t})\right) \equiv\left(\begin{array}{c}
\left(G_{11} x_{2}\right)(t) \\
\left(G_{21} x_{2}\right)(t) \\
\left(G_{31} x_{2}\right)(t)
\end{array}\right)\left(\begin{array}{l}
y_{11}(t) \\
y_{21}(t) \\
y_{31}(t)
\end{array}\right)
$$

where the functions $y_{11}(t), y_{21}(t)$, and $y_{31}(t)$ must be determined in order to know $\left(G_{11} x_{2}\right)(t),\left(G_{21} x_{2}\right)(t)$ and $\left(G_{31} x_{2}\right)(t)$. By virtue of the equivalence (3.16) and note that when $h_{2}(t)=0$, then $x_{2}(t-r)=x_{2}(t)$ to obtain the functional:

$$
\begin{aligned}
\left\langle z_{1}(t), z_{2}(t)\right\rangle=\int_{0}^{t} f & \left\{\frac{1}{2} x_{1}(t) Q x_{2}(t)+x_{1}(t) H \dot{x}_{2}(t)+\mu C_{1}^{2} x_{1}(t) x_{2}(t)+\mu C_{1} C_{2} x_{1}(t) x_{2}(t-r)\right. \\
& -\mu C_{1} x_{1}(t) \dot{x}_{2}(t)+\mu C_{1} C_{2} x_{1}(t) x_{2}(t-r)+\mu C_{2}^{2} x_{1}(t-r) x_{2}(t-r) \\
& -\mu C_{2} x_{1}(t-r) \dot{x}_{2}(t)-\mu D u_{1}(t) \dot{x}_{2}(t)-\mu C_{1} x_{1}(t) \dot{x}_{2}(t)-\mu C_{2} x_{1}(t-r) \dot{x}_{2}(t) \\
& \left.-\mu D u_{1}(t) \dot{x}_{2}(t)+\mu \dot{x}_{1}(t) \dot{x}_{2}(t)\right\} d t
\end{aligned}
$$

On simplifying (3.21) further, we obtain

$$
\begin{gathered}
\left\langle z_{1}(t), z_{2}(t)\right\rangle=\int_{0}^{t}\left\{x_{1}(t)\left[\left(\frac{1}{2} Q+\mu C_{1}^{2}\right) x_{2}(t)+\left(H-2 \mu C_{1}\right) \dot{x}_{2}(t)+2 \mu C_{1} C_{2} x_{2}(t-r)\right]\right. \\
\left.+x_{1}(t-r)\left[-2 \mu C_{2} \dot{x}_{2}+\mu C_{2}^{2} x_{2}(t-r)\right]+\left[u_{1}(t)(-2 \mu D)+\mu \dot{x}_{1}(t)\right] \dot{x}_{2}(t)\right\} d t
\end{gathered}
$$

But by (3.22), we obtain

$$
\begin{aligned}
\left\langle z_{1}(t), z_{2}(t)\right\rangle=\int_{0}^{t} f & \left\{x_{1}(t)\left[\left(\frac{1}{2} Q+\mu C_{1}^{2}\right) x_{2}(t)+\left(H-2 \mu C_{1}\right) \dot{x}_{2}(t)\right]\right. \\
& +x_{1}(t-r)\left[2 \mu C_{1} C_{2} x_{2}(t)-2 \mu C_{2} \dot{x}_{2}(t)+\mu C_{2}^{2} x_{2}(t-r)\right] \\
& \left.+\left[u_{1}(t)(-2 \mu D)+\mu \dot{x}_{1}(t)\right] \dot{x}_{2}(t)\right\} d t
\end{aligned}
$$

Introducing (3.17) to the second term of the integrand in (3.23) we obtain

$$
\int_{0}^{t_{f}}\left\{x_{1}(t-r)\left[2 \mu C_{1} C_{2} x_{2}(t)-2 \mu C_{2} \dot{x}_{2}(t)+\mu C_{2}^{2} x_{2}(t-r)\right]\right\} d t \text {. }
$$

With $s+r=t,-r=0$, we obtain

$$
\begin{aligned}
= & \int_{-r}^{t_{f}-r}\left\{x_{1}(s)\left[2 \mu C_{1} C_{2} x_{2}(s+r)-2 \mu C_{2} \dot{x}_{2}(s+r)+\mu C_{2}^{2} x_{2}(s)\right]\right\} d s \\
= & \int_{-r}^{0}\left\{x_{1}(s)\left[2 \mu C_{1} C_{2} x_{2}(s+r)-2 \mu C_{2} \dot{x}_{2}(s+r)+\mu C_{2}^{2} x_{2}(s)\right]\right\} d s \\
& +\int_{0}^{t_{f}-r}\left\{x_{1}(s)\left[2 \mu C_{1} C_{2} x_{2}(s+r)-2 \mu C_{2} \dot{x}_{2}(s+r)+\mu C_{2}^{2} x_{2}(s)\right]\right\} d s \\
= & \int_{-r}^{0}\left\{h_{1}(t)\left[2 \mu C_{1} C_{2} x_{2}(t+r)-2 \mu C_{2} \dot{x}_{2}(t+r)+\mu C_{2}^{2} x_{2}(t)\right]\right\} d t \\
& +\int_{0}^{t_{f}-r}\left\{x_{1}(t)\left[2 \mu C_{1} C_{2} x_{2}(t+r)-2 \mu C_{2} \dot{x}_{2}(t+r)+\mu C_{2}^{2} x_{2}(t)\right]\right\} d t .
\end{aligned}
$$


Since $x_{1}(t)=h_{1}(t)$ for $t \in[-r, 0]$, (3.35) holds. Also, for $t \in\left[0, t_{f}-r\right]$, let us define $w_{2}(t)=\left\{\begin{array}{c}x_{2}(t+r) ; 0 \leq t \leq t_{f}-r \\ 0 ; t_{f}-r \leq t \leq t_{f}\end{array}\right\}$

Then, we shall be able to keep track of the domain of definition of the function $x_{2}(t+r)$ and the functional $\left\langle z_{1}(t), z_{2}(t)\right\rangle$ of (3.23) may be expressed as follows:

$$
\begin{aligned}
\left\langle z_{1}(t), z_{2}(t)\right\rangle= & \int_{0}^{t_{f}}\left\{x_{1}(t)\left[\left(\frac{1}{2} Q+\mu C_{1}^{2}\right) x_{2}(t)+\left(H-2 \mu C_{1}\right) \dot{x}_{2}(t)\right]+h_{1}(t)\left[\begin{array}{c}
2 \mu C_{1} C_{2} x_{2}(t+r) \\
-2 \mu C_{2} \dot{x}_{2}(t+r)+\mu C_{2}^{2} x_{2}(t)
\end{array}\right]\right. \\
& +x_{1}(t)\left[2 \mu C_{1} C_{2} x_{2}(t+r)-2 \mu C_{2} \dot{x}_{2}(t+r)+\mu C_{2}^{2} x_{2}(t)\right] \\
& \left.+\left[u_{1}(t)(-2 \mu D)+\mu \dot{x}_{1}(t)\right] \dot{x}_{2}(t)\right\} d t
\end{aligned}
$$

Imbedding (3.27) into (3.28), we obtain the expression

$\left\langle z_{1}(t), z_{2}(t)\right\rangle=\int_{0}^{t_{f}}\left\{\begin{array}{c}x_{1}(t)\left[\left(\frac{1}{2} Q+\mu C_{1}^{2}\right) x_{2}(t)+\left(H-2 \mu C_{1}\right) \dot{x}_{2}(t)+2 \mu C_{1} C_{2} w_{2}(t)-2 \mu C_{2} \dot{w}_{2}(t)\right. \\ +\mu C_{2}^{2} x_{2}(t)+h_{1}(t)\left[2 \mu C_{1} C_{2} x_{2}(t+r)-2 \mu C_{2} \dot{x}_{2}(t+r)+\mu C_{2}^{2} x_{2}(t)\right] \\ +\left[u_{1}(t)(-2 \mu D)+\mu \dot{x}_{1}(t)\right] \dot{x}_{2}(t)\end{array}\right\} d t$

$\left\langle z_{1}(t), z_{2}(t)\right\rangle=\int_{0}^{t_{f}}\left\{\begin{array}{c}x_{1}(t)\left[\left(\frac{1}{2} Q+\mu C_{1}^{2}\right) x_{2}(t)+\left(H-2 \mu C_{1}\right) \dot{x}_{2}(t)+2 \mu C_{1} C_{2} w_{2}(t)-2 \mu C_{2} \dot{w}_{2}(t)\right. \\ +\mu C_{2}^{2} x_{2}(t)+h_{1}(t)\left[2 \mu C_{1} C_{2} x_{2}(t+r)-2 \mu C_{2} \dot{x}_{2}(t+r)+\mu C_{2}^{2} x_{2}(t)\right] \\ +u_{1}(t)(-2 \mu D) \dot{x}_{2}(t)+\mu \dot{x}_{1}(t) \dot{x}_{2}(t)\end{array}\right\} d t$

$\left\langle z_{1}(t), z_{2}(t)\right\rangle=\int_{0}^{t}\left\{x_{1}(t) y_{11}(t)+\dot{x}_{1}(t) \dot{y}_{11}(t)+u_{1}(t) y_{21}(t)+h_{1}(t) y_{31}(t)\right\} d t$.

The quantities $y_{11}(t), y_{21}(t)$ and $y_{31}(t)$ which satisfy (3.30) have to be determined. Towards this, let

$\alpha(t)=\left(\frac{1}{2} Q+\mu C_{1}^{2}\right) x_{2}(t)+\left(H-2 \mu C_{1}\right) \dot{x}_{2}(t)+2 \mu C_{1} C_{2} w_{2}(t)-2 \mu C_{2} \dot{w}_{2}(t)$

$\beta(t)=\mu \dot{x}_{2}(t)$

then, $\alpha(t)-y_{11}(t)$ and $\beta(t)-\dot{y}_{11}(t)$ are continuous functions on $\left[0, t_{f}\right]$ and for $x_{1}(.) \in G_{1}\left[0, t_{f}\right]$ such that $x_{1}(0)=0=x_{1}\left(t_{f}\right)$, we have

$$
\int_{0}^{t_{f}}\left\{x_{1}(t)\left[\alpha(t)-y_{11}(t)\right]+\dot{x}_{1}(t)\left[\beta(t)-\dot{y}_{11}(t)\right]\right\} d t=0
$$

so that by Lemma $1, \beta(t)-\dot{y}_{11}(t)$ is differentiable on $\left[0, t_{f}\right]$ with

$$
\frac{d}{d t}\left[\beta(t)-\dot{y}_{11}(t)\right]=\alpha(t)-y_{11}(t) .
$$

This implies that

$$
\ddot{y}_{11}(t)-y_{11}(t)=\dot{\beta}(t)-\alpha(t)
$$

Using (3.32) and (3.33) in (3.36), we obtain

$$
\begin{gathered}
\ddot{y}_{11}(t)-y_{11}(t)=\mu \ddot{x}_{2}(t)-\left[\left(\frac{1}{2} Q+\mu C_{1}^{2}\right) x_{2}(t)+\left(H-2 \mu C_{1}\right) \dot{x}_{2}(t)+2 \mu C_{1} C_{2} w_{2}(t)\right. \\
\left.-2 \mu C_{2} \dot{w}_{2}(t)\right] .
\end{gathered}
$$

Solving the second order differential equation (3.37) for $y_{11}(t)$ and the resulting constants therein eliminated, we obtain the following tidier form:

$$
\begin{aligned}
y_{11}(t)=- & \mu \dot{x}(0) \operatorname{Sinh}\left(t_{f}\right)+\int_{0}^{t_{f}} \mu \dot{x}(s) \operatorname{Sinh}\left(t_{f}-s\right) d s-\int_{0}^{t_{f}} \operatorname{Sinh}\left(t_{f}-s\right) \\
& {\left[\left(\frac{1}{2} Q+\mu C_{1}^{2}+C_{2}^{2}\right) x(s)+\left(H-2 \mu C_{1}\right) \dot{x}(s)+2 \mu C_{1} C_{2} x(s+r)\right.} \\
& \left.-2 \mu C_{2} \dot{x}(s+r)\right] d s+\left[\left(\frac{1}{2} Q+\mu C_{1}^{2}+C_{2}^{2}\right) x(0)+\left(H-2 \mu C_{1}\right) \dot{x}(0)\right. \\
& \left.+2 \mu C_{1} C_{2} x(r)-2 \mu C_{2} \dot{x}(s+r)\right] \operatorname{Cosh}(t)+\frac{\operatorname{Sinht}}{\operatorname{Sinht}_{f}}\left\{\left(\frac{1}{2} Q+\mu C_{1}^{2}+C_{2}^{2}\right) x\left(t_{f}\right)\right. \\
& +\left(H-2 \mu C_{1}\right) \dot{x}\left(t_{f}\right)+2 \mu C_{1} C_{2} x\left(t_{f}-r\right)-2 \mu C_{2} \dot{x}\left(t_{f}-r\right) \\
& -\left[\left(\frac{1}{2} Q+\mu C_{1}^{2}+C_{2}^{2}\right) x(0)+\left(H-2 \mu C_{1}\right) \dot{x}(0)+2 \mu C_{1} C_{2} x(r)\right. \\
& \left.-2 \mu C_{2} \dot{x}(r)\right] \operatorname{Cosh}\left(t_{f}\right)+\int_{0}^{t_{f}} \operatorname{Sinh}\left(t_{f}-s\right)\left[\left(\frac{1}{2} Q+\mu C_{1}^{2}+C_{2}^{2}\right) x(s)\right. \\
& \left.+\left(H-2 \mu C_{1}\right) \dot{x}(s)+2 \mu C_{1} C_{2} x(s+r)-2 \mu C_{2} \dot{x}(s+r)\right] d s \\
& \left.+\mu \dot{x}(0) \operatorname{Sinh}\left(t_{f}\right)-\int_{0}^{t_{f}} \mu \dot{x}(s) \operatorname{Cosh}\left(t_{f}-s\right) d s\right\} ; \quad 0 \leq t \leq t_{f}
\end{aligned}
$$

Now from (3.31), we readily obtain 
Thus the first column operators $\left(G_{11} x_{2}\right)(t),\left(G_{21} x_{2}\right)(t)$ and $\left(G_{31} x_{2}\right)(t)$ are uniquely determined by virtue of (3.20). Repeating the same arguments we obtain the second column of the operator, $\left(G_{12} x_{2}\right)(t),\left(G_{22} x_{2}\right)(t)$ and $\left(G_{32} x_{2}\right)(t)$, setting $x_{2}(t) \equiv h_{2}(t)=0$, then $\dot{x}_{2}(t)=0=x_{2}(t-r)$, and the last column of the operator, $\left(G_{13} x_{2}\right)(t),\left(G_{23} x_{2}\right)(t)$ and $\left(G_{33} x_{2}\right)(t)$, setting $x_{2}(t) \equiv u_{2}(t)=0$, for $0 \leq t \leq t_{f}$. This implies that, $x_{2}(t)=0$ and $x_{2}(t-r)=h_{2}(t)$. With this, we conclude the proof of Theorem 3.1.

\section{Conclusion:}

It follows from here that, while [5] constructed an operator for CLRP, [2] focuses on same class of optimal control problem but with delay parameter in the state variable. The construction of this control operator, G, helps to bridge the gap between Bolza problems and CLRP with delay parameter. This makes the construction of the operator very important and relevant in that, it takes cares of all the variations in CLRP with or without delay parameter in the state variable.

Based on this, our next paper shall be devoted to the numerical application of this operator to CGM algorithm in solving Mayer, Lagrange and Bolza form of Continuous-Time Linear Regulator Problems with or without delay parameter in the state variable with more light on the construction of the Control Operator.

\section{References}

[1] Athans, M. and Falb, P. L., (1966), Optimal Control: An Introduction to the Theory and Its Applications, McGraw-Hill, New York.

[2] Aderibigbe, F. M., (1993), "An Extended Conjugate Gradient Method Algorithm For Control Systems with Delay-I, Advances in Modeling \& Analysis, C, AMSE Press, Vol. 36, No. 3, pp 51-64.

[3] George M. Siouris, (1996), An Engineering Approach To Optimal Control And Estimation Theory, John Wiley \& Sons, Inc., 605 Third Avenue, New York, 10158-00 12.

[4] Hasdorff, L. (1976), Gradient optimization and Nonlinear Control. J. Wiley and Sons, New York.

[5] Ibiejugba, M. R. and Onumanyi, P., (1984), “A Control Operator and some of its Applications, J. Math. Anal. Appl. Vol. 103, No. 1, Pp. 31-47.

[6] David, G. Hull, (2003), Optimal Control Theory for Applications, Mechanical Engineering Series, Springer-Verlag, New York, Inc., 175 Fifth Avenue, New York, NY 10010.

[7] Gelfand, I. M. and Fomin, S. V., (1963), Calculus of Variations, Patience Hall, Inc., New Jersey.

[8] George, F. Simmons, (1963), Introduction to Topology and Modern Analysis, McGraw- Hill Kogakusha, Book Company, Inc., Tokyo, Japan. 